March 2018

\title{
MELAS: a complex and challenging diagnosis
}

Kumail Khandwala

Aga Khan University, kumail.khandwala@aku.edu

Anwar Ahmad

Aga Khan University, anwar.ahmed@aku.edu

Taha Sheikh

Dow University of Health Sciences, Karachi.

Follow this and additional works at: https://ecommons.aku.edu/pakistan_fhs_mc_radiol Part of the Radiology Commons

\section{Recommended Citation}

Khandwala, K., Ahmad, A., Sheikh, T. (2018). MELAS: a complex and challenging diagnosis. Journal of the College of Physicians and Surgeons--Pakistan, 28(3), S46-S48.

Available at: https://ecommons.aku.edu/pakistan_fhs_mc_radiol/106 


\title{
MELAS: A Complex and Challenging Diagnosis
}

\author{
Kumail Khandwala1 , Anwar Ahmed ${ }^{1}$ and Taha Sheikh²
}

\begin{abstract}
Mitochondrial encephalopathy, lactic acidosis and stroke-like episodes (MELAS) is a rare multisystem disorder and is the most common maternally inherited mitochondrial disease. This condition has a special predilection for the nervous system and muscles. Typical findings on brain imaging include stroke-like areas, calcification of basal ganglia and brain atrophy. This accounts for the disease being, both clinically and radiologically, mistaken for ischemic stroke. The differentiation features from stroke include comparatively young age of the patients, site of the lesions, and relative overlap between the cerebral vasculature territories. In this case report, we discuss a 16-year male with clinical and radiological features highly suggestive of MELAS syndrome. Since this disease is rare and its clinical presentation is complex, it is among the most challenging to diagnose. It is particularly difficult to differentiate between ischemic stroke and MELAS. Magnetic resonance imaging (MRI) with diffusion weighted imaging (DWI), susceptibility weighted imaging (SWI) sequences and MR spectroscopy may aid in establishing the diagnosis. A particularly characteristic feature of MELAS syndrome is that recurrence may occur in locations different than previously noted, which was also seen in our patient.
\end{abstract}

Key Words: MELAS. Stroke-like lesions. MRI. Mitochondrial disease.

\section{INTRODUCTION}

Mitochondrial encephalopathy, lactic acidosis and stroke-like episodes (MELAS) is the most common maternally inherited mitochondrial disease with multisystem involvement and a special predilection for the nervous system and muscles. Up to $80 \%$ of cases show a common defect located at nucleotide 3243 of the mitochondrial DNA (mtDNA) gene owing to a point mutation involving transposition from $A$ to $G$, which encodes for transfer RNA (tRNA) for leucine. ${ }^{1}$

Abnormalities involving tRNA affect multiple parts of the respiratory chain. These are hypothesized to cause abnormal protein production throughout the mitochondria. Therefore, the body shifts its functions to derive energy primarily from anaerobic metabolism. This manifests as accumulation of lactic acid, depletion of Nicotinamide Adenine Dinucleotide (NAD) stores causing predisposition to hypoxic injury, resulting in death of neurons in the cortex. This is believed to be the basic pathogenesis of the disease. ${ }^{2,3}$

\section{CASE REPORT}

A 16-year male patient was initially admitted to our hospital because of non-specific abdominal pain, headache, nausea, vomiting and focal seizures. His

${ }^{1}$ Department of Radiology, The Aga Khan University Hospital, Karachi.

2 Medical Student, Dow University of Health Sciences, Karachi.

Correspondence: Dr. Kumail Khandwala, Department of

Radiology, The Aga Khan University Hospital, Stadium Road,

P. O. Box 3500, Karachi-74800.

E-mail: kumail.khandwala@gmail.com

Received: April 20, 2017; Accepted: October 23, 2017. initial neurological examination was normal. A study of cerebrospinal fluid (CSF) samples excluded neuroinfection. Electroencephalogram (EEG) was normal. Computed tomography (CT) scan showed areas of hypoattenuation in bilateral cerebellar hemispheres, raising the suspicion of ischemic changes (Figure 1A). MRI was performed, which revealed areas of high signal on DWI and no significant dropout on Apparent Diffusion Coefficient (ADC) mapping in the cerebellar hemispheres (Figures $1 \mathrm{~B}$ and $\mathrm{C}$ ). The patient was managed on the basis of subacute cerebellar infarcts.

Subsequently, a year later, the patient presented to the Emergency Department with seizures, headache and vomiting. This time the neurological exammation revealed a right-sided visual field defect and decreased visual acuity. EEG showed left temporo-occipital sharp and slow waves with diffuse theta and delta slowing. Extended diagnostic tests were performed. Thyroid antibodies and autoimmune workups were negative. CSF studies revealed a leukocyte count of 2 and an increased lactate level of $4.8 \mathrm{mmol} / \mathrm{L}$. Herpes simplex polymerase chain reaction (PCR) and gene xpert for mycobacterium tuberculosis were negative. Homocysteine levels were normal and workup for porphyria was negative. Left deltoid muscle biopsy showed no evidence of degenerative, regenerative changes, atrophy or inflammation or ragged-red fibers.

MRI was done which showed gyral thickening of the left parieto-occipital cortex extending to the temporal lobe and areas of abnormal, high signal on FLAIR and T2 images with relative loss of cortico-subcortical differentiation, and reduction of signal intensity in these areas on T1 images representing gyral edema (Figures $2 \mathrm{~A}$ and $\mathrm{B}$ ) and (Figures $3 \mathrm{~B}$ and $\mathrm{C}$ ). This time, there was diffusion restriction in these lesions on DWI and 


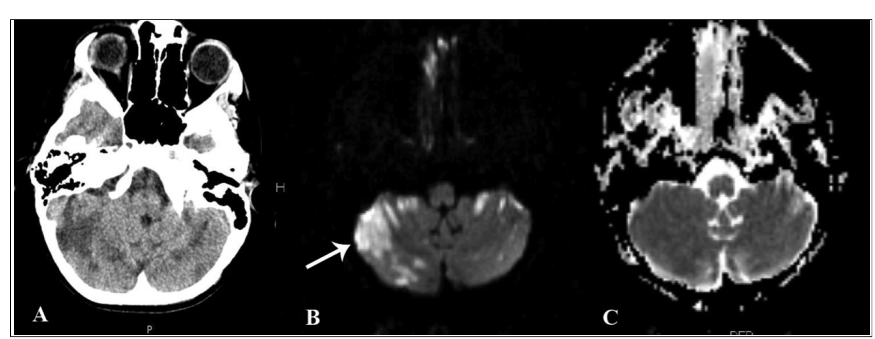

Figure 1: (A) Initial CT scan revealed hypoattenuating areas in bilateral cerebellar hemispheres which were suggestive of ischemic infarcts (B) MRI showed high signal in the cerebellar hemispheres on DWI (C) (white arrow) without any significant dropout on ADC maps.

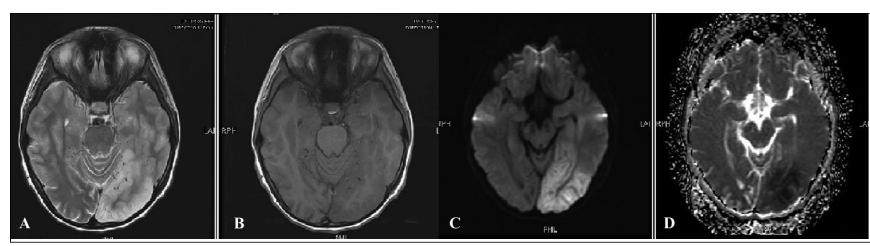

Figure 2: T2, T1 images of MR brain done a year later revealed signal abnormality in the left parieto-occipital lobe along with diffusion restriction in this area on DWI and dropout on ADC mapping.

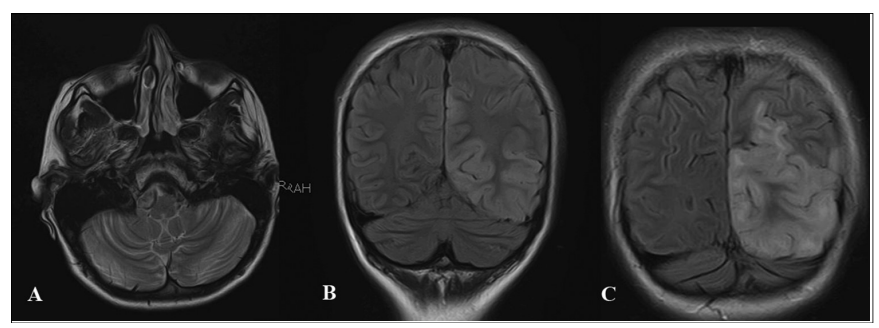

Figure 3: ( $A$ and $B$ ) Previously noted stroke like lesions in the cerebellar hemispheres had almost completely resolved on this examination ( $B$ and $C$ ) FLAIR images revealing gyriform edema and subcortical involvement in the left parietal and occipital lobes.

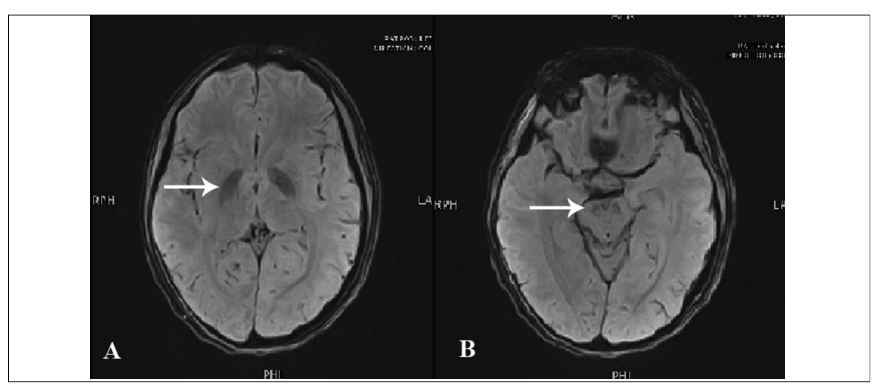

Figure 3: SWI images showing signal dropout in bilateral globus pallidus, substantia nigra and red nuclei (white arrows).

corresponding decrease in cortical signal intensity on ADC maps (Figures 2C and D).

The previously noted stroke-like areas in bilateral cerebellar hemispheres had, however, resolved (Figures $3 A$ and $B$ ). Increased signals were noted on susceptibility weighted imaging (SWI) in bilateral globus pallidus, red nuclei and substantia nigra, which were likely representing iron deposition/calcifications (Figure 4). The whole clinical scenario, investigations carried out during hospitalization, information from history-taking, and CT and MR images of the brain with recurrent, migratory stroke-like episodes were all highly suggestive of MELAS syndrome.

\section{DISCUSSION}

MELAS is a rare multisystem disorder and is the most common maternally inherited mitochondrial disease having a special predilection for the nervous system and muscles. Presentation of patients with this mitochondrial disorder is usually delayed until childhood after a period of normal development, owing to time taken for metabolite build-up and exhaustion of cell's coping mechanism. Most patients, before the age of 40 , have history of multiple stroke-like episodes. These recurrent stroke-like episodes progressively cause widespread damage leading to loss of cognitive mechanism. Characteristic signs and symptoms may include muscle weakness, recurrent headache, weakness and even, vision loss. Patients develop increased anion gap metabolic acidosis from lactic acid buildup that presents as vomiting, abdominal pain, loss of bowel control, fatigue, muscle weakness and breathing difficulty. MELAS syndrome may also present with ataxia and myoclonus as well as endocrine system abnormalities like diabetes mellitus, hypoparathyroidism and hypogonadism. 4

The stroke-like areas are non-vascular and linked to mitochondrial mutation leading to oxidative phosphorylation dysfunction, which subsequently causes encephalopathy. The combination of lactic acidosis, multiple nonvascular strokes, encephalopathic psychosis, diabetes, and sensory neuronal hearing loss cause severe dysfunction leading to increased mental disabilities, physical disabilities, and eventually death. Death may also occur due to cardiac failure, pulmonary embolism, or renal failure.

Typical findings on brain imaging include stroke-like areas, calcification of basal ganglia, and diffuse brain atrophy. ${ }^{5}$ In $90 \%$ of patients with MELAS, the focal neurological symptoms are well correlated with strokelike lesions in the corresponding brain areas. 6 Hypodense areas resembling infarcts are seen on CT imaging. This accounts for the disease being, both clinically and radiologically, mistaken for ischemic stroke initially. The differentiation features from stroke include comparatively young age of the patients, site of the lesions (parietal, temporal and occipital cortices are especially prone), and relative overlap between the cerebral vasculature territories. ${ }^{7}$

The stoke-like areas in MR studies manifest as enlarged gyri with enhanced T2 signals. Subcortical white matter involvement is not unusual. Previous reports have suggested that DWI and ADC sequences prove extremely helpful in distinguishing between stroke-like lesions of MELAS and ischemic stroke. The stroke-like lesions in MELAS syndrome, show increased signal on 
DWI (T2 shine through) with minimal or no change on $A D C$ because these lesions are probably due to vasogenic edema rather than cytotoxic edema, as seen in ischemic stroke. Cytotoxic edema causes diffusion restriction with corresponding dropout on ADC mapping. 7,8 This finding was observed in our patient on the initial MRI, revealing the cerebellar stroke-like lesions. They did not show any significant dropout on ADC mapping. However, dropout on ADC was observed in our case in the subsequent MRI with lesions in the parieto-occipital lobe, suggesting that this feature is not always characteristic. Kim et al. reported that increased, normal and decreased ADC values were found in an equal number of patients with MELAS, suggesting that cytotoxic edema gradually evolves following an acute stroke-like episode in such patients, and this may overlap with hyper-perfusion and vasogenic edema. ${ }^{9}$

Lesions in the subacute phase may sometimes show contrast enhancement due to increased permeability resulting from congestion or reperfusion or as a result of blood-brain barrier damage.5,9 Imminent improvement is seen in the clinical picture as these stroke-like lesions subside with passing time. A particularly characteristic feature of MELAS syndrome is that recurrence may occur in locations different from previously noted, which was also seen in our patient.5,10,11 MR spectroscopy is an additional tool that may reveal elevated lactate peaks, suggesting disturbed anaerobic processes. ${ }^{7,8}$

The differential diagnoses of MELAS syndrome, besides ischemic stroke, includes vasculitides bearing resemblance to Moyamoya and Kawasaki diseases, viral encephalitis, status epilepticus, Creutzfeldt-Jakob disease, Wilson's disease, hypoxia, and other mitochondrial disorders such as Leigh's disease, Kearns-Sayre Syndrome, and myoclonic epilepsy with ragged-red fibers. ${ }^{5}$ This condition is usually associated with a poor prognosis and outcome, as there is no effective treatment for MELAS syndrome established, hence the resultant high morbidity and mortality rates.

In summary, we report a patient with clinical and radiological picture of MELAS syndrome. Since this disease is rare and its clinical presentation is complex, it is among the most challenging to diagnose. It is particularly difficult to differentiate between ischemic stroke and MELAS syndrome. MRI and MR spectroscopy may aid in establishing the diagnosis; however, the radiological features are often non-specific and may overlap. It is also important to consider the other differential diagnoses.

\section{REFERENCES}

1. Goto Y, Nonaka I, Horai S. A mutation in the tRNA (Leu) (UUR) gene associated with the MELAS subgroup of mitochondrial encephalomyopathies. Nature 1990; 348:651-3.

2. Oguro $\mathrm{H}$, lijima $\mathrm{K}$, Takahashi $\mathrm{K}$, et al. Successful treatment with succinate in a patient with MELAS. Intern Med 2004; 43:427-31.

3. Kim HS, Kim DI, Lee BI. Diffusion-weighted image and MR spectroscopic analysis of a case of MELAS with repeated attacks. Yonsei Med J 2001; 42:128-33.

4. Chae JH, Hwang H, Lim BC, Cheong HI, Hwang YS, Kim KJ. Clinical features of $A 3243 G$ mitochondrial tRNA mutation. Brain Dev 2004; 26:459-62.

5. Kim IO, Kim JH: Mitochondrial myopathy-encephalopathylactic-acidosis-and-strokelike episodes (MELAS) syndrome: $\mathrm{CT}$ and MR findings in seven children. AJR Am J Roentgenol 1996; 166:641-5.

6. Goto Y, Horai S, Matsuoka T: Mitochondrial myopathy, encephalopathy, lactic acidosis, and stroke-like episodes (MELAS): a correlative study of the clinical features and mitochondrial DNA mutation. Neurology 1992; 42: 545-50.

7. Abe K, Yoshimura H, Tanaka H. Comparison of conventional and diffusion-weighted MRI and proton MR spectroscopy in patients with mitochondrial encephalomyopathy, lactic acidosis, and stroke-like events. Neuroradiology, 2004; 46: 113-7.

8. Yoneda M, Maeda M, Kimura H. Vasogenic edema in MELAS: a serial study with diffusion-weighted MR imaging. Neurology. 1999; 53: 2182-84

9. Kim JH, Lim MK, Jeon TY. Diffusion and perfusion characteristics of MELAS (Mitochondrial myopathy, encephalopathy, lactic acidosis, and stroke-like episode) in thirteen patients. Korean $J$ Radiol 2011; 12:15-24.

10. Hasuo K, Tamura S, Yasumon K. Computed tomography and angiography in MELAS (mitochondrial myopathy, encephalopathy, lactic acidosis and stroke-like episodes); report of 3 cases. Neuroradiology 1987; 29:393-7.

11. Allard JC, Tilak S, Carter AP. CT and MR of MELAS syndrome. Am J Neuroradiol 1988; 9:234-38. 